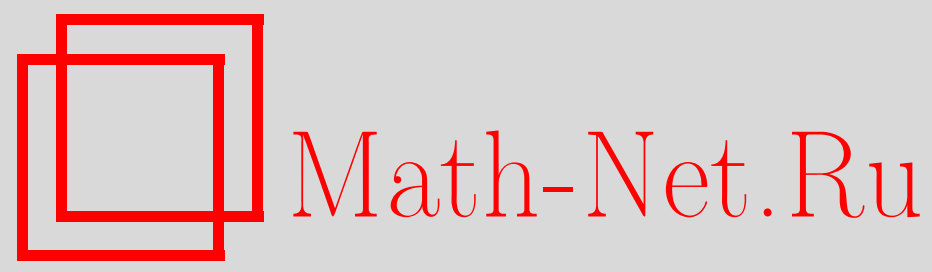

P. Х. Садикова, Общий класс неравенств со смешанными средними, Матем. заметки, 1997, том 61, выпуск 6, 864872

DOI: https://doi.org/10.4213/mzm1570

Использование Общероссийского математического портала Math-Net.Ru подразумевает, что вы прочитали и согласны с пользовательским соглашением http://www. mathnet.ru/rus/agreement

Параметры загрузки:

IP : 3.81 .55 .215

26 апреля 2023 г., 15:25:48

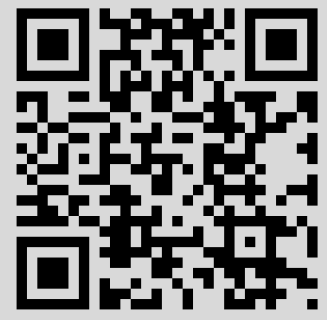




\section{ОБЩИЙ КЛАСС НЕРАВЕНСТВ СО СМЕШАННЫМИ СРЕДНИМИ}

\section{P.X. Садикова}

Пусть $(T, \Sigma, \mu)$ - пространство с положительной мерой, $f: \mathbb{R} \rightarrow \mathbb{R}$ - строго монотонная непрерывная функция, $\mathfrak{G}(T)$ - совокупность вещественных $\mu$-измеримых функций на $T$. Пусть $x(\cdot) \in \mathfrak{G}(T)$ и $(f \circ x)(\cdot) \in L_{1}(T, \mu)$. Для средних $\mathfrak{M}_{(T, \mu, f)}(x(\cdot))$ и смешанных средних $\mathfrak{M}_{\left(T_{1}, \mu_{1}, f_{1}\right)}\left(\mathfrak{M}_{\left(T_{2}, \mu_{2}, f_{2}\right)}(x(\cdot))\right)$ доказаны сравнения, из которых выводятся аналоги или обобщения некоторых классических неравенств Гёльдера, Минковского, Беллмана, Пирсона, Годуновой и Левина, Стеффенсена, Маршалла и Олкина и др.

Результаты являются продолжением исследований автора.

Библиография: 14 названий.

Предварительные сведения. Пусть $(T, \Sigma, \mu)$ - пространство с положительной мерой, $f: \mathbb{R} \rightarrow \mathbb{R}$ - строго монотонная непрерьвная функция и $\mathfrak{G}(T)$ - совокупность вещественньх $\mu$-измеримых функций на $T$. Пусть $x(\cdot) \in \mathfrak{G}(T)$ и $(f \circ x)(\cdot) \in L_{1}(T, \mu)$. Обозначим

$$
\begin{gathered}
\mathfrak{M}_{(T, \mu, f)}(x(\cdot))=f^{-1}\left(\int_{T} f(x(t)) d \mu(t)\right), \\
L_{f}=\left\{x(\cdot) \in \mathfrak{G}(T) \mid \mathfrak{M}_{(T, \mu, f)}(x(\cdot))<\infty\right\}, \quad \mathfrak{M}_{(T, \mu, f)}: L_{f} \rightarrow \mathbb{R} .
\end{gathered}
$$

Для функции $x(\cdot) \in \mathfrak{G}(T)$, где $T=T_{1} \times \cdots \times T_{n}$, положим

$$
\begin{aligned}
& \mathfrak{M}_{\left(T_{1}, \mu_{1}, f_{1}\right), \ldots,\left(T_{n}, \mu_{n}, f_{n}\right)}(x(\cdot)) \\
& \quad=\left(\mathfrak{M}_{\left(T_{n}, \mu_{n}, f_{n}\right)} \circ \mathfrak{M}_{\left(T_{n-1}, \mu_{n-1}, f_{n-1}\right)} \circ \cdots \circ \mathfrak{M}_{\left(T_{1}, \mu_{1}, f_{1}\right)}\right)(x(\cdot)), \\
& L_{f_{1}, \ldots, f_{n}}=\left\{x(\cdot) \in \mathfrak{G}(T) \mid \mathfrak{M}_{\left(T_{1}, \mu_{1}, f_{1}\right), \ldots,\left(T_{n}, \mu_{n}, f_{n}\right)}(x(\cdot))<\infty\right\} .
\end{aligned}
$$

Вообще говоря,

$$
\mathfrak{M}_{\left(T_{1}, \mu_{1}, f_{1}\right),\left(T_{2}, \mu_{2}, f_{2}\right)}(x(\cdot)) \neq \mathfrak{M}_{\left(T_{2}, \mu_{2}, f_{2}\right),\left(T_{1}, \mu_{1}, f_{1}\right)}(x(\cdot)) .
$$

Отметим два свойства, которые будут использованы в дальнейшем:

a) если $x_{1}(\cdot) \leqslant x_{2}(\cdot)$ почти всюду, то $\mathfrak{M}_{(T, \mu, f)}\left(x_{1}(\cdot)\right) \leqslant \mathfrak{M}_{(T, \mu, f)}\left(x_{2}(\cdot)\right)$;

б) если $f_{1}=f_{2}=\cdots=f_{n}=f$, то

$$
\begin{aligned}
\mathfrak{M}_{\left(T_{1}, \mu_{1}, f\right), \ldots,\left(T_{n}, \mu_{n}, f\right)}(x(\cdot)) & =\mathfrak{M}_{\left(T_{i_{1}}, \mu_{i_{1}}, f\right), \ldots,\left(T_{i_{n}}, \mu_{i_{n}}, f\right)}(x(\cdot)) \\
& =\mathfrak{M}_{(T, f)}(x(\cdot)),
\end{aligned}
$$

где $\left\{i_{1}, \ldots, i_{n}\right\}$ - любая перестановка $(1,2, \ldots, n)$ и $T=T_{1} \times \cdots \times T_{n}$ (это свойство следует из теоремы Фубини). 
Доказаны общие теоремы для таких смешанных средних, из которых выводятся аналоги или обобщения некоторых классических неравенств [1]-[7]. Результаты обобщают исследования автора [8]-[12].

При выводе частных случаев общих результатов будут использованы теоремы Иессена [1, с. 96], Шиша и Карго [13], Купера [14].

Теорема (Иессен). Пусть $(T, \Sigma, \mu)$ - пространство с неотрицательной конечной мерой $\mu, f_{1}, f_{2}$ - непрерывные строго монотонные функции, $f_{2}$ возрастает (m.е. $f_{2}^{-1}$ возрастает), $f_{2} \circ f_{1}^{-1}$ выпукла. Тогда для функиии $x(\cdot): T \rightarrow \mathbb{R}$ имеет место неравенство

$$
f_{1}^{-1}\left(\frac{1}{\mu(T)} \int_{T} f_{1}(x(t)) d \mu(t)\right) \leqslant f_{2}^{-1}\left(\frac{1}{\mu(T)} \int_{T} f_{2}(x(t)) d \mu(t)\right) .
$$

ЗАмЕЧАнИЕ. Вьпуклость $f_{2} \circ f_{1}^{-1}$ гарантирует, например, следующее предположение о функциях $f_{1}, f_{2}$ : функция $x(\cdot) \mapsto f_{2}^{\prime}(x(\cdot)) / f_{1}^{\prime}(x(\cdot)), f_{1}^{\prime}(x(\cdot)) \neq 0 \forall x(\cdot) \in T$

1) не убывает при одинаковой монотонности $f_{1}, f_{2}$, или

2) не возрастает при различной монотонности $f_{1}, f_{2}$.

При этом предположении неравенство Иессена доказано Шиша и Карго [13].

Теорема (Купер). Пусть $f_{1}, f_{2}$ - строго монотонные непрерывные функиии и выполняется одно из условий:

1) $f_{1}$ убиввет, $f_{2}$ возрастает, или

2) обе функиии $f_{1}, f_{2}$ убывают либо возрастают, при этом функиия $\left(f_{1} / f_{2}\right)(x)$ возрастает, $f_{2}(x) \neq 0$.

Тогда для любых $x_{1}, \ldots, x_{n}$ справедливо неравенство

$$
f_{1}^{-1}\left(\sum_{i=1}^{n} f_{1}\left(x_{i}\right)\right) \leqslant f_{2}^{-1}\left(\sum_{i=1}^{n} f_{2}\left(x_{i}\right)\right) .
$$

Равенство имеет место в случае 1) и, если $f_{1} / f_{2}$ строго возрастает, в случае 2$)$, только тогда, когда $x_{i} \in \mathbb{R}_{+}$для некоторого $i$.

\section{Общие результаты.}

Теорема 1. Пусть $\left(T_{i}, \Sigma_{i}, \mu_{i}\right), i=1,2,-$ пространства с положительными мерами; $f_{j}, j=1,2,3,4,-$ строго монотонные непрерывные функиии на $\mathbb{R}$, при этом

$$
\begin{array}{ll}
\mathfrak{M}_{\left(T_{1}, \mu_{1}, f_{1}\right)}(x(\cdot)) \leqslant \mathfrak{M}_{\left(T_{1}, \mu_{1}, f_{2}\right)}(x(\cdot)) \leqslant \mathfrak{M}_{\left(T_{1}, \mu_{1}, f_{3}\right)}(x(\cdot)) & \forall x(\cdot) \in \mathfrak{G}\left(T_{1}\right), \\
\mathfrak{M}_{\left(T_{2}, \mu_{2}, f_{2}\right)}(x(\cdot)) \leqslant \mathfrak{M}_{\left(T_{2}, \mu_{2}, f_{4}\right)}(x(\cdot)) & \forall x(\cdot) \in \mathfrak{G}\left(T_{2}\right) .
\end{array}
$$

Тогда для любого $x(\cdot) \in \mathfrak{G}\left(T_{1} \times T_{2}\right)$ имеют место неравенства

$$
\begin{aligned}
& \mathfrak{M}_{\left(T_{1}, \mu_{1}, f_{1}\right),\left(T_{2}, \mu_{2}, f_{2}\right)}(x(\cdot)) \leqslant \mathfrak{M}_{\left(T_{2}, \mu_{2}, f_{4}\right),\left(T_{1}, \mu_{1}, f_{3}\right)}(x(\cdot)), \\
& \mathfrak{M}_{\left(T_{2}, \mu_{2}, f_{2}\right),\left(T_{1}, \mu_{1}, f_{1}\right)}(x(\cdot)) \leqslant \mathfrak{M}_{\left(T_{1}, \mu_{1}, f_{3}\right),\left(T_{2}, \mu_{2}, f_{4}\right)}(x(\cdot)) .
\end{aligned}
$$

В теореме 1 функции $f_{1}, f_{2}$ зависимы. Докажем теорему, в которой функции $f_{1}, f_{2}$ независимы, что позволяет расширить область применимости общих результатов. 
Теорема 2. Пусть $\left(T_{i}, \Sigma_{i}, \mu_{i}\right), i=1,2$, - пространства с положсительными мерами $\mu_{i} ; f_{j}, j=1,2,3,4,-$ строго монотонные непрерывные функиии на $\mathbb{R}$, при этом

$$
\begin{array}{ll}
\mathfrak{M}_{\left(T_{1}, \mu_{1}, f_{1}\right)}(x(\cdot)) \leqslant \mathfrak{M}_{\left(T_{1}, \mu_{1}, f_{3}\right)}(x(\cdot)) \leqslant \mathfrak{M}_{\left(T_{1}, \mu_{1}, f_{4}\right)}(x(\cdot)) & \forall x(\cdot) \in \mathfrak{G}\left(T_{1}\right), \\
\mathfrak{M}_{\left(T_{2}, \mu_{2}, f_{2}\right)}(x(\cdot)) \leqslant \mathfrak{M}_{\left(T_{2}, \mu_{2}, f_{3}\right)}(x(\cdot)) & \forall x(\cdot) \in \mathfrak{G}\left(T_{2}\right) .
\end{array}
$$

Тогда для любого $x(\cdot) \in \mathfrak{G}\left(T_{1} \times T_{2}\right)$ имеют место неравенства

$$
\begin{aligned}
& \mathfrak{M}_{\left(T_{1}, \mu_{1}, f_{1}\right),\left(T_{2}, \mu_{2}, f_{2}\right)}(x(\cdot)) \leqslant \mathfrak{M}_{\left(T_{2}, \mu_{2}, f_{3}\right)\left(T_{1}, \mu_{1}, f_{4}\right)}(x(\cdot)) \\
& \mathfrak{M}_{\left(T_{2}, \mu_{2}, f_{2}\right),\left(T_{1}, \mu_{1}, f_{1}\right)}(x(\cdot)) \leqslant \mathfrak{M}_{\left(T_{1}, \mu_{1}, f_{4}\right),\left(T_{2}, \mu_{2}, f_{3}\right)}(x(\cdot))
\end{aligned}
$$

Путем повторного применения результатов $(3),(4),(7),(8)$ можем получить сравнения обобщенных средних для многомерного случая.

ТеОрема 3. Пусть $\left(T_{i}, \Sigma_{i}, \mu_{i}\right), i=1, \ldots, n,-$ пространства с положсительными мерами $\mu_{i} ; f_{i}, f_{i}^{\prime}, i=1, \ldots, n, u \varphi$ - строго монотонные непрерывные функиии на $\mathbb{R}, \varphi$ возрастающая, $I(u)=u$, при этом

$$
\begin{aligned}
\mathfrak{M}_{\left(T_{i}, \mu_{i}, f_{i}\right)}(x(\cdot)) \leqslant \mathfrak{M}_{\left(T_{i}, \mu_{i}, \varphi\right)}(x(\cdot)), & i=1, \ldots, n, & \forall x(\cdot) \in \mathfrak{G}\left(T_{i}\right), \\
\mathfrak{M}_{\left(T_{i}, \mu_{i}, I\right)}(x(\cdot)) \leqslant \mathfrak{M}_{\left(T_{i}, \mu_{i}, f_{i}^{\prime}\right)}(x(\cdot)), & i=1, \ldots, n, & \forall x(\cdot) \in \mathfrak{G}\left(T_{i}\right) .
\end{aligned}
$$

Тогда для всякого $x(\cdot) \in \mathfrak{G}\left(T_{1} \times \cdots \times T_{n}\right)$ имеет место неравенство

$$
\mathfrak{M}_{\left(T_{1}, \mu_{1}, f_{1}\right), \ldots,\left(T_{n}, \mu_{n}, f_{n}\right)}(x(\cdot)) \leqslant \varphi^{-1}\left(\mathfrak{M}_{\left(T_{i_{1}}, \mu_{i_{1}}, f_{i_{1}}^{\prime}\right), \ldots,\left(T_{i_{n}}, \mu_{i_{n}}, f_{i_{n}}^{\prime}\right)}(\varphi(x(\cdot)))\right)
$$

әде $\left(i_{1}, \ldots, i_{n}\right)$ - любая перестановка $(1, \ldots, n)$, а также справедливо неравенство

$$
\mathfrak{M}_{\left(T_{1}, \mu_{1}, f_{1}\right), \ldots,\left(T_{n}, \mu_{n}, f_{n}\right)}(x(\cdot)) \geqslant \varphi^{-1}\left(\mathfrak{M}_{\left(T_{i_{1}}, \mu_{i_{1}}, f_{i_{1}}^{\prime}\right), \ldots,\left(T_{i_{n}}, \mu_{i_{n}}, f_{i_{n}}^{\prime}\right)}(\varphi(x(\cdot)))\right)
$$

в случае, если условия (9), (10) противоположсного смысла.

Общие теоремы 1-3 и их следствия могут быть применены к доказательству новых неравенств.

ЛЕмма (следствие из теоремы 1$)$. Пусть $\left(T_{1}, \Sigma_{1}, \mu_{1}\right)$ - пространство с вероятностной мерой, $\left(T_{2}, \Sigma_{2}, \mu_{2}\right)$ - пространство с положительной мерой, $f_{1}, f_{2}-$ строго монотонные непрерывные функиии на $\mathbb{R}, f_{1}$ вогнута, $f_{2}$ выпукла, $I(u)=u$, при этом

$$
\mathfrak{M}_{\left(T_{1}, \mu_{1}, f_{1}\right)}(x(\cdot)) \leqslant \mathfrak{M}_{\left(T_{1}, \mu_{1}, I\right)}(x(\cdot)) \leqslant \mathfrak{M}_{\left(T_{1}, \mu_{1}, f_{2}\right)}(x(\cdot)) \quad \forall x(\cdot) \in \mathfrak{G}\left(T_{1}\right) .
$$

Тогда для любого $x(\cdot) \in \mathfrak{G}\left(T_{1} \times T_{2}\right)$ имеют место неравенства

$$
\begin{aligned}
& \mathfrak{M}_{\left(T_{1}, \mu_{1}, f_{1}\right)}\left(\mathfrak{M}_{\left(T_{2}, \mu_{2}, I\right)}(x(\cdot))\right) \leqslant \mathfrak{M}_{\left(T_{2}, \mu_{2}, I\right)}\left(\mathfrak{M}_{\left(T_{1}, \mu_{1}, f_{2}\right)}(x(\cdot))\right) \\
& \mathfrak{M}_{\left(T_{2}, \mu_{2}, I\right)}\left(\mathfrak{M}_{\left(T_{1}, \mu_{1}, f_{1}\right)}(x(\cdot))\right) \leqslant \mathfrak{M}_{\left(T_{1}, \mu_{1}, f_{2}\right)}\left(\mathfrak{M}_{\left(T_{2}, \mu_{2}, I\right)}(x(\cdot))\right)
\end{aligned}
$$

Сформулированная лемма используется при вьводе обобщений неравенств Пирсона-Стеффенсена и при доказательстве следующей теоремы. 
Теорема 4. Пусть $\left(T_{1}, \Sigma_{1}, \mu_{1}\right)$ - пространство с вероятностной мерой, $\left(T_{1}, \Sigma_{1}, \nu_{1}\right),\left(T_{2}, \Sigma_{2}, \mu_{2}\right)$ - пространства с положительными мерами и выполнены условия леммы (т.е. $f_{1}$ - вогнутая, $f_{2}$ - выпуклая строго монотонные непрерывные функиии). Пусть, далее, $K(\cdot, \cdot): T_{1} \times T_{2} \rightarrow \mathbb{R}_{+}-$измеримая функиия, $K(\cdot, \cdot) \in L_{1}\left(T_{1} \times T_{2}, \nu_{1} \times \mu_{2}\right), K\left(t_{1}, \cdot\right) \in L_{1}\left(T_{2}, \mu_{2}\right) \quad \forall t_{1} \in T_{1}$, а функиия $x(\cdot)$ допускает представление

$$
x\left(t_{1}\right)=\int_{T_{2}} K\left(t_{1}, t_{2}\right) d \mu_{2}\left(t_{2}\right) \quad \forall t_{1} \in T_{1} .
$$

Если $\forall t_{2} \in T_{2}$ имеют место соотношения

$$
\mathfrak{M}_{\left(T_{1}, \mu_{1}, f_{2}\right)}\left(K\left(\cdot, t_{2}\right)\right) \leqslant \mathfrak{M}_{\left(T_{1}, \nu_{1}, I\right)}\left(K\left(\cdot, t_{2}\right)\right) \leqslant \mathfrak{M}_{\left(T_{1}, \mu_{1}, t_{1}\right)}\left(K\left(\cdot, t_{2}\right)\right)
$$

то для $x(\cdot) \in \mathfrak{G}\left(T_{1}\right)$ справедливы неравенства

$$
\mathfrak{M}_{\left(T_{1}, \mu_{1}, f_{1}\right)}(x(\cdot)) \leqslant \mathfrak{M}_{\left(T_{1}, \nu_{1}, I\right)}(x(\cdot)) \leqslant \mathfrak{M}_{\left(T_{1}, \mu_{1}, f_{2}\right)}(x(\cdot))
$$

Равенство в (14) достигается только при $x(\cdot)=$ const.

ДокаЗАТЕЛЬСтво теоремы 1. Докажем неравенства (3), а неравенства (4) доказываются аналогично. Последовательно применяя свойства а), б) и неравенства (1), (2), получим

$$
\begin{aligned}
& \mathfrak{M}_{\left(T_{1}, \mu_{1}, f_{1}\right),\left(T_{2}, \mu_{2}, f_{2}\right)}(x(\cdot))=\mathfrak{M}_{\left(T_{2}, \mu_{2}, f_{2}\right)}\left(\mathfrak{M}_{\left(T_{1}, \mu_{1}, f_{1}\right)}(x(\cdot))\right) \\
& \quad \leqslant \mathfrak{M}_{\left(T_{2}, \mu_{2}, f_{2}\right)}\left(\mathfrak{M}_{\left(T_{1}, \mu_{1}, f_{2}\right)}(x(\cdot))\right)=\mathfrak{M}_{\left(T_{1}, \mu_{1}, f_{2}\right)}\left(\mathfrak{M}_{\left(T_{2}, \mu_{2}, f_{2}\right)}(x(\cdot))\right) \\
& \quad \leqslant \mathfrak{M}_{\left(T_{1}, \mu_{1}, f_{3}\right)}\left(\mathfrak{M}_{\left(T_{2}, \mu_{2}, f_{2}\right)}(x(\cdot))\right) \leqslant \mathfrak{M}_{\left(T_{1}, \mu_{1}, f_{3}\right)}\left(\mathfrak{M}_{\left(T_{2}, \mu_{2}, f_{4}\right)}(x(\cdot))\right) \\
& \quad=\mathfrak{M}_{\left(T_{2}, \mu_{2}, f_{4}\right),\left(T_{1}, \mu_{1}, f_{3}\right)}(x(\cdot)),
\end{aligned}
$$

что и требовалось.

ДоказАтельство теоремы 2. Докажем неравенства (7), а неравенства (8) доказьваются аналогично. Исходя из свойств а), б) и неравенств $(5),(6)$, имеем

$$
\begin{aligned}
& \mathfrak{M}_{\left(T_{1}, \mu_{1}, f_{1}\right),\left(T_{2}, \mu_{2}, f_{2}\right)}(x(\cdot))=\mathfrak{M}_{\left(T_{2}, \mu_{2}, f_{2}\right)}\left(\mathfrak{M}_{\left(T_{1}, \mu_{1}, f_{1}\right)}(x(\cdot))\right) \\
& \quad \leqslant \mathfrak{M}_{\left(T_{2}, \mu_{2}, f_{2}\right)}\left(\mathfrak{M}_{\left(T_{1}, \mu_{1}, f_{3}\right)}(x(\cdot))\right) \leqslant \mathfrak{M}_{\left(T_{2}, \mu_{2}, f_{3}\right)}\left(\mathfrak{M}_{\left(T_{1}, \mu_{1}, f_{3}\right)}(x(\cdot))\right) \\
& \quad=\mathfrak{M}_{\left(T_{1}, \mu_{1}, f_{3}\right)}\left(\mathfrak{M}_{\left(T_{2}, \mu_{2}, f_{3}\right)}(x(\cdot))\right) \leqslant \mathfrak{M}_{\left(T_{1}, \mu_{1}, f_{4}\right)}\left(\mathfrak{M}_{\left(T_{2}, \mu_{2}, f_{3}\right)}(x(\cdot))\right) \\
& \quad=\mathfrak{M}_{\left(T_{2}, \mu_{2}, f_{3}\right),\left(T_{1}, \mu_{1}, f_{4}\right)}(x(\cdot)),
\end{aligned}
$$

что доказьвает (7). 
ДОКАЗАТЕЛЬСТВО ТЕОРЕМЫ 3. В силу обозначений, свойств а), б) и неравенств (9), (10), имеем

$$
\begin{aligned}
\mathfrak{M}_{\left(T_{1}, \mu_{1}, f_{1}\right), \ldots,\left(T_{n}, \mu_{n}, f_{n}\right)}(x(\cdot)) \\
\quad=\mathfrak{M}_{\left(T_{n}, \mu_{n}, f_{n}\right)}\left(\mathfrak{M}_{\left(T_{n-1}, \mu_{n-1}, f_{n-1}\right)}\left(\ldots\left(\mathfrak{M}_{\left(T_{1}, \mu_{1}, f_{1}\right)}(x(\cdot))\right) \ldots\right)\right) \\
\quad \leqslant \mathfrak{M}_{\left(T_{n}, \mu_{n}, \varphi\right)}\left(\mathfrak{M}_{\left(T_{n-1}, \mu_{n-1}, f_{n-1}\right)}\left(\ldots\left(\mathfrak{M}_{\left(T_{1}, \mu_{1}, f_{1}\right)}(x(\cdot))\right) \ldots\right)\right) \\
\quad \leqslant \mathfrak{M}_{\left(T_{n}, \mu_{n}, \varphi\right)}\left(\mathfrak{M}_{\left(T_{n-1}, \mu_{n-1}, \varphi\right)}\left(\ldots\left(\mathfrak{M}_{\left(T_{1}, \mu_{1}, f_{1}\right)}(x(\cdot))\right) \ldots\right)\right) \leqslant \ldots \\
\quad \leqslant \mathfrak{M}_{\left(T_{n}, \mu_{n}, \varphi\right)}\left(\mathfrak{M}_{\left(T_{n-1}, \mu_{n-1}, \varphi\right)}\left(\ldots\left(\mathfrak{M}_{\left(T_{1}, \mu_{1}, \varphi\right)}(x(\cdot))\right) \ldots\right)\right)=\mathfrak{M}_{(T, \varphi)}(x(\cdot)) \\
\quad=\mathfrak{M}_{\left(T_{i_{1}}, \mu_{i_{1}}, \varphi\right), \ldots,\left(T_{i_{n}}, \mu_{i_{n}}, \varphi\right)}(x(\cdot))=\varphi^{-1}\left(\int_{T_{i_{1}}} \ldots \int_{T_{i_{n}}} \varphi\left(x\left(t_{1}, \ldots, t_{n}\right)\right) d \mu_{i_{n}} \ldots d \mu_{i_{1}}\right) \\
\quad \leqslant \varphi^{-1}\left(\mathfrak{M}_{\left(T_{i_{1}}, \mu_{i_{1}}, f_{i_{1}}^{\prime}\right)}\left(\int_{T_{i_{2}}} \ldots \int_{T_{i_{n}}} \varphi\left(x\left(t_{1}, \ldots, t_{n}\right)\right) d \mu_{i_{n}} \ldots d \mu_{i_{1}}\right)\right) \leqslant \ldots \\
\quad \leqslant \varphi^{-1}\left(\mathfrak{M}_{\left(T_{i_{1}}, \mu_{i_{1}}, f_{i_{1}}^{\prime}\right)}\left(\mathfrak{M}_{\left(T_{i_{2}}, \mu_{i_{2}}, f_{i_{2}}^{\prime}\right)}\left(\ldots\left(\mathfrak{M}_{\left(T_{i_{n}}, \mu_{i_{n}}, f_{i_{n}}^{\prime}\right)}(\varphi(x(\cdot))) \ldots\right)\right)\right)\right. \\
\quad=\varphi^{-1}\left(\mathfrak{M}_{\left(T_{i_{1}}, \mu_{i_{1}}, f_{i_{1}}^{\prime}\right), \ldots,\left(T_{i_{n}}, \mu_{i_{n}}, f_{i_{n}}^{\prime}\right)}(\varphi(x(\cdot))),\right.
\end{aligned}
$$

что и требовалось.

ДокАЗАТЕЛЬСТво ЛЕммЫ. Пусть в теореме $2\left(T_{1}, \Sigma_{1}, \mu_{1}\right)$ - пространство с вероятностной мерой, $f_{2}(u)=f_{3}(u)=u=I(u)$, при этом условие (5) обрашается в равенство, а условие (6) вьполняется согласно неравенству Иессена (т.е. $f_{1}$ вогнута, $f_{4}=f_{2}$ вьпукла, заменяем функцию $f_{4}$ на $\left.f_{2}\right)$. Из общих результатов $(7),(8)$ соответственно следуют неравенства (11), (12).

ДоКАЗАТЕЛЬСТВО ТЕОРЕМЫ 4. Все части дВойного неравенства (13) проинтегрируем по множеству $T_{2}$ с мерой $\mu_{2}$ :

$$
\begin{aligned}
\int_{T_{2}} f_{2}^{-1}\left(\int_{T_{1}}\left(K\left(t_{1}, t_{2}\right)\right) d \mu_{1}\left(t_{1}\right)\right) d \mu_{2}\left(t_{2}\right) & \leqslant \int_{T_{2}}\left(\int_{T_{1}} K\left(t_{1}, t_{2}\right) d \nu_{1}\left(t_{1}\right)\right) d \mu_{2}\left(t_{2}\right) \\
& \leqslant \int_{T_{2}} f_{1}^{-1}\left(\int_{T_{1}} f_{1}\left(K\left(t_{1}, t_{2}\right)\right) d \mu_{1}\left(t_{1}\right)\right) d \mu_{2}\left(t_{2}\right) .
\end{aligned}
$$

В средней части неравенства поменяем порядок интегрирования (условия теоремы Фубини, очевидно, выполнены), к правой и левой частям применим соответственно доказанные в лемме оценки (12) и (11). Получим

$$
\begin{aligned}
& f_{1}^{-1}\left(\int_{T_{1}} f_{1}\left(\int_{T_{2}} K\left(t_{1}, t_{2}\right) d \mu_{2}\left(t_{2}\right)\right) d \mu_{1}\left(t_{1}\right)\right) \leqslant \int_{T_{1}}\left(\int_{T_{2}} K\left(t_{1}, t_{2}\right) d \mu_{2}\left(t_{2}\right)\right) d \nu_{1}\left(t_{1}\right) \\
& \quad \leqslant f_{2}^{-1}\left(\int_{T_{1}} f_{2}\left(\int_{T_{2}} K\left(t_{1}, t_{2}\right) d \mu_{2}\left(t_{2}\right)\right) d \mu_{1}\left(t_{1}\right)\right) .
\end{aligned}
$$

В силу представления для функции $x\left(t_{1}\right)$ имеем

$$
f_{1}^{-1}\left(\int_{T_{1}} f_{1}\left(x\left(t_{1}\right)\right) d \mu_{1}\left(t_{1}\right)\right) \leqslant \int_{T_{1}} x\left(t_{1}\right) d \nu_{1}\left(t_{1}\right) \leqslant f_{2}^{-1}\left(\int_{T_{1}} f_{2}\left(x\left(t_{1}\right)\right) d \mu_{1}\left(t_{1}\right)\right)
$$


откуда следует

$$
\mathfrak{M}_{\left(T_{1}, \mu_{1}, f_{1}\right)}(x(\cdot)) \leqslant \mathfrak{M}_{\left(T_{1}, \nu_{1}, I\right)}(x(\cdot)) \leqslant \mathfrak{M}_{\left(T_{1}, \mu_{1}, f_{2}\right)}(x(\cdot)) .
$$

Общий результат (14) является неравенством той же природы, что и неравенство Стеффенсена [6], а также включает в себя известную лемму Пирсона [2, с. 170]. Убедимся в этом. Рассмотрим следствия из теоремы 4.

СлЕДСТВИЕ 1. Пусть в теореме 4 ядро функиии $x\left(t_{1}\right)$ представимо в виде

$$
K\left(t_{1}, t_{2}\right)= \begin{cases}1, & t_{2} \in T_{\alpha}, \quad T_{\alpha} \subset T_{2} \\ 0, & t_{2} \notin T_{\alpha}\end{cases}
$$

Тогда

$$
x\left(t_{1}\right)=\int_{T_{\alpha}} 1 \cdot d \mu_{2}\left(t_{2}\right) \quad \forall t_{1} \in T_{1}
$$

Правая часть условия (13) при таком предположении преобразуется следующим образом:

$$
\int_{T_{1}} 1 \cdot d \nu_{1}\left(t_{1}\right) \leqslant f_{2}^{-1}\left(\int_{T_{1}} f_{1}(1) d \mu_{1}\left(t_{1}\right)\right)
$$

т.е. $\nu_{1}\left(T_{1}\right) \leqslant \mu_{1}\left(T_{1}\right)$, или можем положить $\nu_{1}\left(T_{1}\right)=\mu_{1}\left(T_{1}^{\prime}\right), T_{1}^{\prime} \subset T_{1}$.

При перечисленных условиях правая часть общего неравенства (14) примет вид

$$
\int_{T_{1}} x(t) d \nu_{1}(t)=\int_{T_{1}^{\prime}} x(t) d \mu_{1}(t) \leqslant f_{2}^{-1}\left(\int_{T_{1}^{\prime}} f_{2}(x(t)) d \mu_{1}(t)\right), \quad T_{1}^{\prime} \subset T_{1}
$$

или при $d \nu_{1}(t)=y(t) d \mu_{1}(t), 0 \leqslant y(t) \leqslant 1 \forall t \in T_{1}$, имеет место неравенство

$$
\int_{T_{1}} x(t) y(t) d \mu_{1}(t) \leqslant f_{2}^{-1}\left(\int_{T_{1}^{\prime}} f_{2}(x(t)) d \mu_{1}(t)\right), \quad T_{1}^{\prime} \subset T_{1}
$$

Итак, неравенство справедливо для функций $x(\cdot)$, представимых в виде

$$
x(t)=\int_{T_{\alpha}} d \mu_{2}\left(t_{2}\right), \quad T_{\alpha} \subset T_{2} \quad \forall t \in T_{1},
$$

при выполнении условия $\mu_{1}\left(T_{1}^{\prime}\right)=\nu_{1}\left(T_{1}\right)=a$, иными словами,

$$
\int_{T_{1}} d \nu_{1}(t)=\int_{T_{1}^{\prime}} y(t) d \mu_{1}(t)=a, \quad 0 \leqslant y(t) \leqslant 1
$$

для вьпуклой функции $f_{2}$ и $\left(f_{2} \circ x\right)(\cdot) \in L_{1}\left(T_{1}^{\prime}, \mu_{1}\right)$.

Из неравенства (14) при $f_{2}(u)=u$ следует лемма Пирсона. Приведем ее. 
Лемма (Пирсон [2, с. 170]). Пусть $(T, \Sigma, \mu)$ - пространство с положительной мерой $\mu, x(\cdot) \geqslant 0, x(\cdot) \in L_{1}(T, \mu), T_{\alpha} \subset T, T_{\alpha}=\{t \mid x(t) \geqslant \alpha\}, \mu\left(T_{\alpha}\right)=a$, $0 \leqslant y(t) \leqslant 1 \quad \forall t \in T$,

$$
\int_{T} y(t) d \mu(t)=a
$$

Тогда

$$
\int_{T} x(t) y(t) d \mu(t) \leqslant \int_{T_{\alpha}} x(t) d \mu(t)
$$

СлЕДСтвиЕ 2. Пусть в теореме $4 T_{1}=T_{2}=[0 ; a]$, ядро в представлении $x(\cdot)$ равно

$$
K\left(t_{1}, t_{2}\right)=\left\{\begin{array}{ll}
1, & t_{2} \leqslant t_{1}, \\
0, & t_{2}>t_{1}
\end{array} \quad \forall t_{1} \in[0 ; a]\right.
$$

m.e.

$$
x\left(t_{1}\right)=\int_{0}^{t_{1}} 1 \cdot d \mu_{2}(t)
$$

Пусть $d \mu_{1}(t)=d t, d \nu_{1}(t)=y(t) d t, 0 \leqslant y(t) \leqslant 1 \quad \forall t \in[0 ; a]$. Тогда из условия (13) $\forall t \in[0 ; a]$ имеем

$$
\int_{0}^{t} y(\tau) d \tau \leqslant f_{1}^{-1}\left(\int_{0}^{t} f_{1}(\tau) d \tau\right)
$$

откуда

$$
\int_{0}^{t} y(\tau) d \tau \leqslant \int_{0}^{t} d \tau
$$

$\Pi p u t=a$ получаем

$$
\int_{0}^{a} y(\tau) d \tau \leqslant a,
$$

а из общего неравенства (14) (правая часть) следует

$$
\int_{0}^{a} x(t) y(t) d t \leqslant f_{2}^{-1}\left(\int_{0}^{c} f_{2}(x(t)) d t\right), \quad \text { əде } \quad c=\int_{0}^{a} y(t) d t
$$

или

$$
\int_{0}^{a} x(t) Y^{\prime}(t) d t \leqslant f_{2}^{-1}\left(\int_{0}^{Y(a)} f_{2}(x(t)) d t\right), \quad Y(t)=\int_{0}^{t} y(\tau) d \tau .
$$

Последняя оценка включает в себя неравенства Стеффенсена $\left(f_{2}(u)=u\right)[6]$, Беллмана $\left(f_{2}(u)=u^{p}, p \geqslant 1\right)\left[2\right.$, с. 75], Годуновой $\left(f_{2}(u)=u^{p}, p \geqslant 1\right)[3]$, Пекарича $\left(f_{2}(u)=u^{p}\right.$, $p \geqslant 1)[5]$ для более широкого, чем у названных авторов, класса функций $x(\cdot)$ :

$$
x(\cdot)=\int_{0}^{t} d \mu_{2}(t) \quad \forall t \in[0 ; a],
$$

где $\mu_{2}$ - положительная мера, $x(\cdot) \in L_{p}\left([0 ; a], \mu_{2}\right)$ и

$$
\int_{0}^{t} y(\tau) d \tau \leqslant t \quad \forall t \in[0 ; a]
$$

вместо того, что $x(\cdot)$ убывающая и $0 \leqslant y(t) \leqslant 1 \forall t \in[0 ; a]$. 
Теорема (Пекарич). Пусть $f:[0 ; 1] \rightarrow \mathbb{R}_{+}-$невозрастающая функция, $g:[0 ; 1] \rightarrow \mathbb{R}_{+}, 0 \leqslant g(t) \leqslant 1 \quad \forall t \in[0 ; 1]$. Если $p \geqslant 1, \lambda=\left(\int_{0}^{1} g(t) d t\right)^{p}$, mo

$$
\left(\int_{0}^{1} g(t) f(t) d t\right)^{p} \leqslant \int_{0}^{\lambda}(f(t))^{p} d t
$$

$u$

$$
\left(\int_{0}^{1} g(t) f(t) d t\right)^{p} / \int_{0}^{1} g(t) d t \leqslant \frac{1}{\lambda} \int_{0}^{\lambda} f(t) d t
$$

Некоторые частные случаи общих неравенств. Покажем, что общие результаты включают классические неравенства и другие известные оценки. Применим теорему 1. Пусть $\left(T_{2}, \Sigma_{2}, \mu_{2}\right)$ - пространство с конечной положительной мерой, а пространство $\left(T_{1}, \Sigma_{1}, \mu_{1}\right)$ состоит из конечного числа точек с мерами $\mu_{1} \geqslant 0, \sum_{i=1}^{n} \mu_{i}=1$. Тогда при $f_{2}(u)=f_{3}(u)=f_{4}(u)=u$, вогнутой $f_{1}$ (условие (1) вьполняется по теореме Иессена, а условие (2) обращается в равенство) из общего неравенства (3) следуют

1) неравенство Гёльдера, если положить $f_{1}(u)=\ln u, x_{i}(t)=\left|y_{i}(t)\right| /\left\|y_{i}(t)\right\|_{L_{1}(T, \mu)}$;

2) неравенство Минковского, если положить $f_{1}(u)=u^{1 / p}, p>1$,

$$
\mu_{i}=\frac{\left\|y_{i}(\cdot)\right\|_{L_{p}(T, \mu)}}{\sum_{i=1}^{n}\left\|y_{i}(\cdot)\right\|_{L_{p}(T, \mu)}}, \quad x_{i}(t)=\frac{\left|y_{i}(t)\right|^{p}}{\left\|y_{i}(\cdot)\right\|_{L_{p}(T, \mu)}} ;
$$

3) неравенство Годуновой, если положить $\left|x_{i}(t)\right|=\left|y_{i}(t)\right| /\left\|y_{i}(\cdot)\right\|_{L_{p}(T, \mu)}$.

Как частный случай теоремы 1 выведем аналог неравенства Минковского для $x(\cdot) \in$ $L_{p p}\left(T_{1} \times T_{2}, \mu_{1} \times \mu_{2}\right):$

$$
\left\|\int_{T_{1}}|x(\cdot)| d \mu_{1}\left(t_{1}\right)\right\|_{L_{p}\left(T_{2}, \mu_{2}\right)}^{p} \leqslant \int_{T_{1}}\|x(\cdot)\|_{L_{p}\left(T_{2}, \mu_{2}\right)}^{p} d \mu_{1}\left(t_{1}\right) .
$$

Он получается, если $\left(T_{1}, \Sigma_{1}, \mu_{1}\right)$ - пространство с вероятностной мерой, $\left(T_{2}, \Sigma_{2}, \mu_{2}\right)$ пространство с конечной положительной мерой $\mu_{2}$ и $f_{1}(u)=u, f_{2}(u)=f_{3}(u)=f_{4}(u)=u^{p}$, $p>1$. При тех жепредположениях условие (1) теоремы 1 справедливо по теореме Иессена, а условие (2) обрашается в равенство, поэтому из общего неравенства (4) имеем (15).

Пусть в теореме $1\left(T_{1}, \Sigma_{1}, \mu_{1}\right)$ - вероятностное пространство, а $\left(T_{2}, \Sigma_{2}, \mu_{2}\right)$ - пространство с конечной положительной мерой, тогда при $f_{2}(u)=f_{3}(u)=f_{4}(u)=u$, $f_{1}(u)=\ln u$ получаем из (3) обобщенное неравенство Гёльдера, приведенное в монографии А. Маршалла и И. Олкина [7, с. 464-465].

Автор благодарен профессору В.М. Тихомирову за обсуждение результатов. 


\section{СПИСОК ЦИТИРОВАННОЙ ЛИТЕРАТУРЫ}

[1] Харди Г., Литтльвуд Дж., Полиа Г. Неравенства. М.: ИЛ, 1948.

[2] Беллман Р., Бекенбах Э. Неравенства. М.: Мир, 1965.

[3] Годунова Е. К. Неравенства с выпуклыми функциями. Дисс. . . к.ф.-м.н. М.: МГПИ, 1965.

[4] Годунова Е. К., Левин В. И. Общий класс неравенств, содержащий неравенство Стеффенсена // Матем. заметки. 1968. Т. 3. №3. С. 339-344.

[5] Pecaric J. On the Bellman generalization of Steffensen's inequality // J. Math. Anal. Appl. 1982. V. 88. № 2. P. 505-507.

[6] Steffensen J. Bounds of certain trigonometric integrals // 10th Scandinavian Math. Congress. 1945. P. 181-186.

[7] Маршалл А., Олкин И. Неравенства: теория мажорации и ее приложения. М.: Мир, 1983.

[8] Садикова Р. Х. Общий класс неравенств, содержащий неравенство Стеффенсена и его обобщения // Изв. вузов. Матем. 1980. № 4. С. 79-80.

[9] Садикова Р. Х. Неравенства с обобщенными средними // Деп. Изв. вузов. Матем. 1981. № 1456.

[10] Садикова Р. Х. Общий класс неравенств с обобщенньми средними // Деп. Изв. вузов. Матем. 1983. № 2631-84.

[11] Садикова Р. Х. Общий класс неравенств с точными константами. Дисс. ... к. ф.-м.н. М.: Университет дружбы народов, 1990.

[12] Садикова Р.X. Сравнение смешанных обобщенных средних // Деп. Изв. вузов. Матем. 1975. № 3439 .

[13] Shisha O., Cargo G. On comparable means // Pacif. J. Math. 1964. V. 4. № 3. P. 1053-1059.

[14] Cooper R. Notes on certain inequalities. I // J. London Math. Soc. 1927. № 2. P. 17-21; II // J. London Math. Soc. 1927. № 2. P. 156-163. 